\title{
Use of Telemedicine Technologies in Diabetes Prevention and Control in Resource-Constrained Settings: Lessons Learned from Emerging Economies
}

\author{
Rajendra Pradeepa, PhD, Ramachandran Rajalakshmi, MBBS, DO, MRCS, PhD, \\ and Viswanathan Mohan, MD, FRCP, PhD, DSc, FACP, FACE, MACP
}

\begin{abstract}
Telemedicine is a promising strategy that utilizes telecommunication to provide health care in remote areas, facilitating beneficial interaction between the health care provider and people in rural areas and making affordable and accessible medical care available to remote, inaccessible areas of the world. This article provides an overview of some of the ways telemedicine is improving diabetes care outcomes at the community level. Telemedicine can play a number of roles in moving quality diabetes care forward. It is currently being used to create awareness among urban and rural population about the risk factors and prevention of diabetes; to facilitate patient monitoring; for remote diabetic retinopathy screening; and in diabetes prevention at the primary, secondary, and tertiary level. We also highlight the use of automated artificial intelligence software combined with telemedicine to conduct efficient real-time screening of complications such as diabetic retinopathy in remote areas where such facilities are currently unavailable.
\end{abstract}

Keywords: Diabetes, Telemedicine, Teleophthalmology, Retinopathy, Rural, Remote.

\section{Introduction}

$\mathbf{T}$ HE GLOBAL PREVALENCE of diabetes continues to increase with an estimated 425 million people currently affected, and this number is estimated to escalate to 629 million by the year $2045 .{ }^{1}$ Over $90 \%$ of all affected individuals have type 2 diabetes. Four out of five people with diabetes live in low- and middle-income countries (LMIC), approximately half of whom remain undiagnosed according to the International Diabetes Federation. ${ }^{1}$

India ranks second after China in the global diabetes epidemic and is expected to surpass China in the next few years. ${ }^{1}$ India also has a large rural population $(>70 \%)$ and the prevalence of diabetes in rural areas is rising rapidly. Tackling rural diabetes has its own challenges, including poverty, illiteracy, lack of accessibility, and nonavailability of essential drugs and specialized care. ${ }^{2,3}$ Moreover, the ratio of undiagnosed to diagnosed diabetes is higher. ${ }^{4}$

The increasing prevalence of diabetes in low-income countries is of great concern because of high morbidity and mortality and the costs associated with the treatment of complications of diabetes. ${ }^{5}$ Among individuals with selfreported diabetes in rural areas of India, for example, good glycemic control, defined as glycated hemoglobin $\left(\mathrm{HbA}_{1 \mathrm{c}}\right)$ $<7 \%$, was observed in only $30.8 \%$ of the $15.4 \%$ of people who reported having checked their $\mathrm{HbA}_{1 \mathrm{c}}$ during the past year. ${ }^{6}$ Thus, in rural areas, diabetes remains a neglected disorder. $^{7}$

Models of care that are tailored to resource-constrained countries are needed, especially in remote rural areas, to provide better access to screening for diabetes and its associated complications as well as offer better management. Several technology-based approaches to reach out to remote and underserved communities and monitor their health at their door step are now being explored.

Recent advances in information technology and widespread availability of the mobile phone present unique opportunities for prevention, control, and management of chronic diseases like diabetes. ${ }^{8}$ These approaches include telemedicine, mobile technology, and use of the internet.

Madras Diabetes Research Foundation and Dr. Mohan's Diabetes Specialties Centre, WHO Collaborating Centre for Non-communicable Diseases Prevention and Control and IDF Centre of Excellence in Diabetes Care, Chennai, India. 
However, there is need for developing comprehensive interventions and integrating available technologies to be able to provide comprehensive care for diabetes. This article provides an overview of the role of telemedicine technologies in improving diabetes care and how we have used it in India.

\section{Telemedicine and Diabetes Care}

Telemedicine is increasingly being utilized in the field of diabetology to improve access to health care, quality of care, and clinical/psychosocial outcomes in people with diabetes. As many aspects of our lives have become automated and addressed online, so will routine medical care of chronic diseases. $^{9}$

Mobile health refers to the use of mobile communication technologies to promote health by supporting health data collection, delivery of health care information, and/or patient observation, provision of care, ${ }^{10}$ and disease prevention. ${ }^{11}$ Although access to this technology has rapidly expanded in developing countries, ${ }^{12}$ its potential benefits in improving health care are still underutilized. Mobile phone messaging applications, including text messaging or short message service (SMS) and multimedia message service, could offer a convenient and cost-effective way to support desirable health behaviors for preventive health care by providing educational and motivational advice. ${ }^{12}$ Several studies have assessed the feasibility of mobile technology to prevent and manage type 2 diabetes. $^{13-18}$

\section{Role of telemedicine in creating awareness}

A large-scale, community-based diabetes awareness and prevention program, prevention awareness counseling, and evaluation (PACE) was initiated to improve awareness about diabetes. ${ }^{14}$ PACE included four components: (1) a free public awareness campaign with lectures and interactive programs; (2) 164 "PACE Education Counters" opened at various bookshops, shopping complexes, food chain stores, and family physician clinics in Chennai (southeastern India), which provided free diabetes educational materials; (3) mass media programs, including a documentary film/radio program on diabetes and diabetes prevention and management messages provided through newspaper articles, cinema theater advertisements, and text messaging on mobile phones; and (4) training for general practitioners in diabetes management and prevention strategies.

Through the PACE diabetes project, a total of 774 screening and education camps were conducted all over Chennai and 76,645 individuals were screened for diabetes. Among those screened, 17\% had known diabetes, $4 \%$ had newly diagnosed diabetes, and $7.5 \%$ had prediabetes. In addition, 150,000 SMS messages were sent to subscribers on prevention of diabetes to general population and management of diabetes throughout the state of Tamil Nadu (southern tip of India). Assessments conducted after the PACE project (in 2007) showed improvements in diabetes awareness and knowledge compared with results of the earlier CURES survey conducted in 2001-2002. ${ }^{19}$ This project is an example of how telemedicine can increase awareness of diabetes. ${ }^{14,19}$

Further evidence of telemedicine efficacy comes from a prospective, parallel-group, randomized controlled trial involving Asian Indian men with impaired glucose tolerance. Conducted at 10 sites, the study assessed whether mobile phone messaging that encouraged lifestyle change could reduce incident type 2 diabetes. ${ }^{18}$ This study reported that the cumulative incidence of type 2 diabetes was lower in the intervention group who received mobile phone messages $(18 \%)$ than in controls $(27 \%)$. Investigators concluded that mobile phone messaging is an effective and acceptable method to deliver advice and support toward lifestyle modification to prevent type 2 diabetes, in men at high risk.

\section{Role of telemedicine in self-monitoring of blood glucose}

The increased burden of diabetes has prompted increasing efforts to develop mobile technologies for monitoring of blood glucose among individuals with diabetes. During the past decade, much progress has been made in utilizing technology to monitor blood glucose levels. Glycemic control in individuals with diabetes can be measured by various means, including laboratory-based glucose monitoring, $\mathrm{HbA}_{1 \mathrm{c}}$, self-monitoring of blood glucose (SMBG), and continuous glucose monitoring system, using ambulatory glucose profiles for data interpretation and analysis.

SMBG is a valuable tool for decision-making for both patients and clinicians. ${ }^{20}$ Regular SMBG has been shown to improve $\mathrm{HbA}_{1 \mathrm{c}}$ and reduce the fear of both hyperglycemia and hypoglycemia in individuals with type 1 and type 2 diabetes. $^{21,22}$ A wide range of blood glucose meters are available that are portable, inexpensive, accurate, and reliable. One of the barriers to frequent and regular SMBG is patients' inability to make decisions based on the results. However, this issue could be addressed by the use of telemedicine technology. The increasing use of smartphones and availability of mobile applications for management of diabetes present a possible method to transmit the SMBG test results to a health care provider electronically through emails, SMS, or Internet portal.

The Structured Testing Program (STeP) study evaluated how a structured patient/physician SMBG intervention influenced the timing, frequency, and effectiveness of primary care physicians' treatment changes among 483 poorly controlled, insulin-naive type 2 diabetes patients over a year. ${ }^{23}$ On assessing the impact of structured SMBG on physician treatment modification recommendations (TMRs), it was reported that patients who received at least one TMR demonstrated a greater reduction in $\mathrm{HbA}_{1 \mathrm{c}}(-1.2 \%)$ than the active control group $(-0.8 \%)$. The STeP study investigators concluded that a collaborative use of structured SMBG data leads to earlier, more frequent, and more effective TMRs.

Quinn et al. ${ }^{24,25}$ have studied mobile-based health applications and improvements in glycemic control. A study conducted in $2008,{ }^{24}$ among 28 patients with type 2 diabetes, showed that the intervention group, which received cell phone-based software, achieved statistically significant improvements in $\mathrm{HbA}_{1 \mathrm{c}}$ compared to the patients in the control group. Another study conducted by the same group reported that, including mobile application coaching and patient/provider web portals to community primary care reduced $\mathrm{HbA}_{1 \mathrm{c}}$ levels in patients with type 2 diabetes compared with those on standard diabetes management. ${ }^{25}$

In the recent 6-month, prospective, multicenter, single-arm study by Mora et al., ${ }^{26}$ participants used a novel SMBG system (Accu-Chek ${ }^{\circledR}$ Connect diabetes management system; 
Roche Diabetes Care, Indianapolis, IN) that automatically transmits blood glucose data from the meter to a smartphone app and then to secure personal and health care professional portals. ${ }^{26}$ The health care portal automatically organizes the glucose data, identifying patients who are at risk for acute glycemic events, which enables clinicians to triage patients according to the greatest need. Results from the study showed a strong association between use of the system and improved glycemic control, increased patient satisfaction, and reduced distress among individuals with insulin-treated diabetes.

Thus, utilizing mobile technology to review blood glucose data could provide an opportunity to reduce glycemic variability and complications associated with diabetes. ${ }^{27}$ Moreover, new technologies are being explored, which eliminate the need for a blood glucose meter, allowing patients to use their smartphone and test strips to obtain a test result and then provide their result through a variety of digital modes to their clinician.

\section{Real-time telemedicine}

Real-time telemedicine is a promising strategy for improving access to care and care delivery in image-oriented fields like ophthalmology and radiology, where diagnostic decisions are often based on review of photographs/images that are captured by trained technicians. ${ }^{28}$

India is home to over 72 million people with diabetes, ${ }^{1}$ all of whom require annual retinal examination for early detection of diabetic retinopathy. However, access to screening is significantly limited. In India, the ophthalmologist-patient ratio in urban India is $1: 25,000$, but in rural India, it is $\sim 1: 250,000 .{ }^{29}$ Reports have shown that $35 \%-80 \%$ of people with diabetes do not follow the eye care recommendations. ${ }^{30}$

Diabetic retinopathy produces no visual or ocular symptoms until the advanced stages of retinopathy are reached. The value of screening is well established, as early detection of diabetic retinopathy helps in timely treatment with modalities that can decrease the risk of severe visual impairment. ${ }^{31}$

Inadequate number of trained optometrists/retina specialists and the high cost of the conventional fundus cameras are major challenges to providing regular screening of the large number of people with diabetes in India. ${ }^{32}$ The inequitable distribution of ophthalmologists/eye hospitals across the country further widens the gap between demand and availability of eye care to people with diabetes in rural areas and places a major burden on the health care system of India. Studies have shown that digital retinal color photography (either mydriatic or nonmydriatic) is a practical alternative for regular screening of people with diabetes for retinopathy. ${ }^{33,34}$

Teleophthalmology is, thus, a promising solution for enabling secure transmission of information and transfer of digital retinal images for diagnosis and decision-making by an ophthalmologist or retinal specialist and timely referral for treatment of diabetic retinopathy when indicated (e.g., laser photocoagulation and intravitreal injections). ${ }^{35,36}$

\section{Guidelines and model for diabetic retinopathy telemedicine program}

The American Telemedicine Association and Diabetic Retinopathy Telehealth Practice Recommendations Working Group $^{37}$ have provided guidelines and recommended that the equipment used for diabetic retinopathy telescreening must conform to the requisites of the local and federal bodies, which specify that the diagnostic accuracy of the fundus camera used for retinal imaging must be validated and the telemedicine technology must adhere to the standards of Digital Imaging and Communication in Medicine. ${ }^{38}$

The four main components of diabetic retinopathy telemedicine screening are as follows: (1) retina image acquisition - at least one posterior pole image of each eye; (2) image review and evaluation by a trained retina specialist/grader; (3) supervision of patient care; and (4) data and retinal image storage and most importantly, timely referral to a retinal specialist when further treatment for diabetic retinopathy is indicated. ${ }^{36}$

Digital retinal fundus photography imaging in teleophthalmology. Conventional stereoscopic fundus cameras remain the gold standard for retinal examinations. However, these cameras are expensive. Recently, digital retinal color photography with smaller cost-effective fundus cameras has been validated for diabetic retinopathy screening. A few examples include the Visuscout handheld camera and Remidio fundus on phone (FOP) camera, which is a smartphonebased imaging device. ${ }^{39}$

The FOP camera provides good quality high-resolution retinal images and has been successfully used in teleophthalmology screening. ${ }^{39}$ These sleek devices are easy to handle and require minimal training for technicians. They have helped reduce the economic burden on the health care system related to screening for diabetic retinopathy. Imaging through teleophthalmology services helps to detect not only diabetic retinopathy but also other important diabetic eye disorders like cataract, glaucoma, and age-related macular degeneration.

Teleophthalmology screening services are offered at Dr. Mohan's Diabetes Specialties Centre, which has a network of 45 diabetes centers across 10 states of India. In 2017, 16,226 individuals with diabetes were screened for diabetic retinopathy. The retinal images taken using Remidio FOP camera were transferred through local area networks and wide area networks to enable remote diabetic retinopathy grading by the ophthalmologists at the main hospital at Chennai (Fig. 1).

Using the International Clinical Classification of Diabetic Retinopathy grading system, ${ }^{40}$ diabetic retinopathy was detected in $3701(22.8 \%)$ individuals, of which 1140 $(7.02 \%)$ had sight-threatening diabetic retinopathy that required referral for further evaluation and treatment. The smartphone-based retinal imaging system, FOP, is reasonably sensitive and specific for diabetic retinopathy detection and, thus, is, useful as an effective screening tool for diabetic retinopathy. ${ }^{39}$

\section{Ophthalmoscopy versus teleophthalmology for diabetic retinopathy screening}

Two important aspects which need to be considered for the sustainability of a rural outreach telescreening program are cost-effectiveness and patient satisfaction levels. Table 1 shows the differences between ophthalmologist assessment versus teleophthalmology-based assessment of diabetic retinopathy. Gomez-Ulla et al. performed a cost analysis for diabetic retinopathy screening by ophthalmoscopy versus teleophthalmology. ${ }^{41}$ Results showed that although ophthalmoscopy was less expensive, compared to the higher initial investment for a teleophthalmology setup, the latter was 

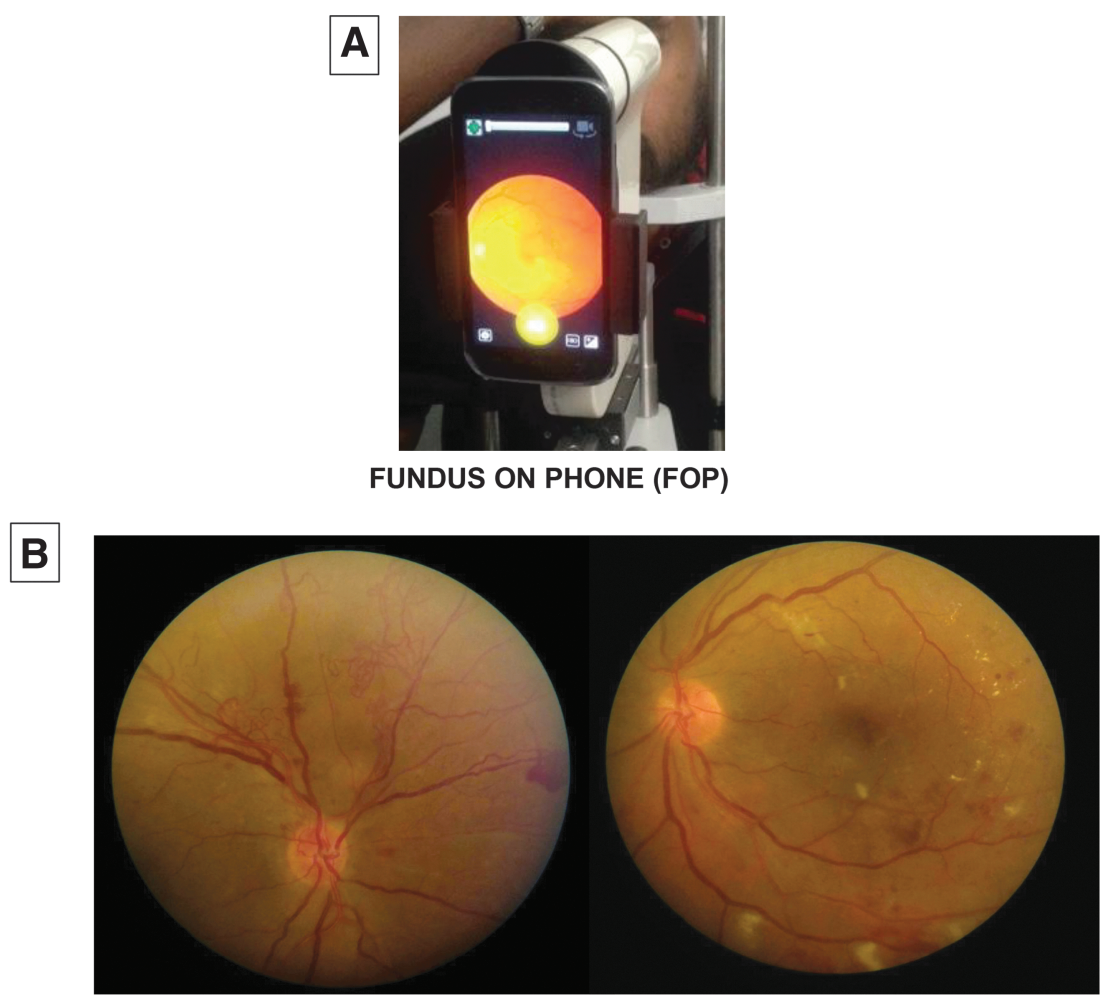

RIGHT EYE: PROLIFERATIVE DIABETIC RETINOPATHY, LEFT EYE: DIABETIC MACULAR EDEMA

FIG. 1. Smartphone-based fundus camera for teleophthalmology, for diabetic retinopathy screening. The figure shows the retinal photographs of a 40-year-old male with type 2 diabetes of 3 -year duration seen at a remote village in southern Tamil Nadu. The patient had no ocular symptoms and a normal vision (6/6) in both eyes. The retinal photographs taken using Remidio FOP camera showed proliferative diabetic retinopathy in the right eye and severe diabetic macular edema in the left eye. After teleconsultation with the ophthalmologist at the base hospital at Chennai-400 km away-regarding his diabetic eye condition and after reviewing his retinal photographs, he was asked to undergo further investigations and treatment for sight-threatening diabetic retinopathy. Early timely detection of diabetic retinopathy by teleophthalmology thus helped this patient to safeguard his vision. FOP, fundus on phone.

found to be more beneficial for the people screened as discussed in Table 1.

One of the possible limitations in a teleophthalmology program is that there is no face-to-face personal interaction with the doctor, with which many patients are most familiar. However, even in telemedicine, the patients do have virtual access to the medical specialist. Some studies have addressed this concern and report excellent patient satisfaction levels $(94 \%)$ with teleophthalmology screening. ${ }^{42,43}$

\section{Artificial intelligence in diabetic retinopathy detection in telemedicine}

The use of artificial intelligence (AI) to analyze retinal images and provide grading of diabetic retinopathy and referral recommendations accurately has generated great interest recently among physicians and other health care personnel. ${ }^{44,45} \mathrm{AI}$ is an appealing concept as it fits in with the current trend of teleophthalmology/telemedicine, and

Table 1. Ophthalmoscopy Versus Telemedicine for Diabetic Retinopathy Screening

\begin{tabular}{|c|c|c|}
\hline & Ophthalmoscopy & Telemedicine \\
\hline Personnel & $\begin{array}{l}\text { Requires trained ophthalmologist/retina } \\
\text { specialist for fundus examination (direct } \\
\text { and indirect ophthalmoscopy) }\end{array}$ & $\begin{array}{l}\text { Retinal photography is taken by trained } \\
\text { technicians, only the grading requires the } \\
\text { trained retina specialist }\end{array}$ \\
\hline $\begin{array}{l}\text { Dilatation of the } \\
\text { pupil (mydriasis) }\end{array}$ & Mandatory & Optional or targeted mydriasis \\
\hline \multirow[t]{3}{*}{ Cost } & Capital expenditure is less & $\begin{array}{l}\text { Capital expenditure toward telescreening } \\
\text { equipment is more }\end{array}$ \\
\hline & Revenue expenditure toward salary is more & Revenue expenditure toward salary is less \\
\hline & Maintenance cost less & Maintenance cost more \\
\hline Cost to patient & More & Less \\
\hline $\begin{array}{l}\text { Convenience to people } \\
\text { in rural areas }\end{array}$ & $\begin{array}{l}\text { More travel, more time consuming and hence } \\
\text { less convenient }\end{array}$ & $\begin{array}{l}\text { Less travel, less time spent, and hence more } \\
\text { convenient }\end{array}$ \\
\hline
\end{tabular}




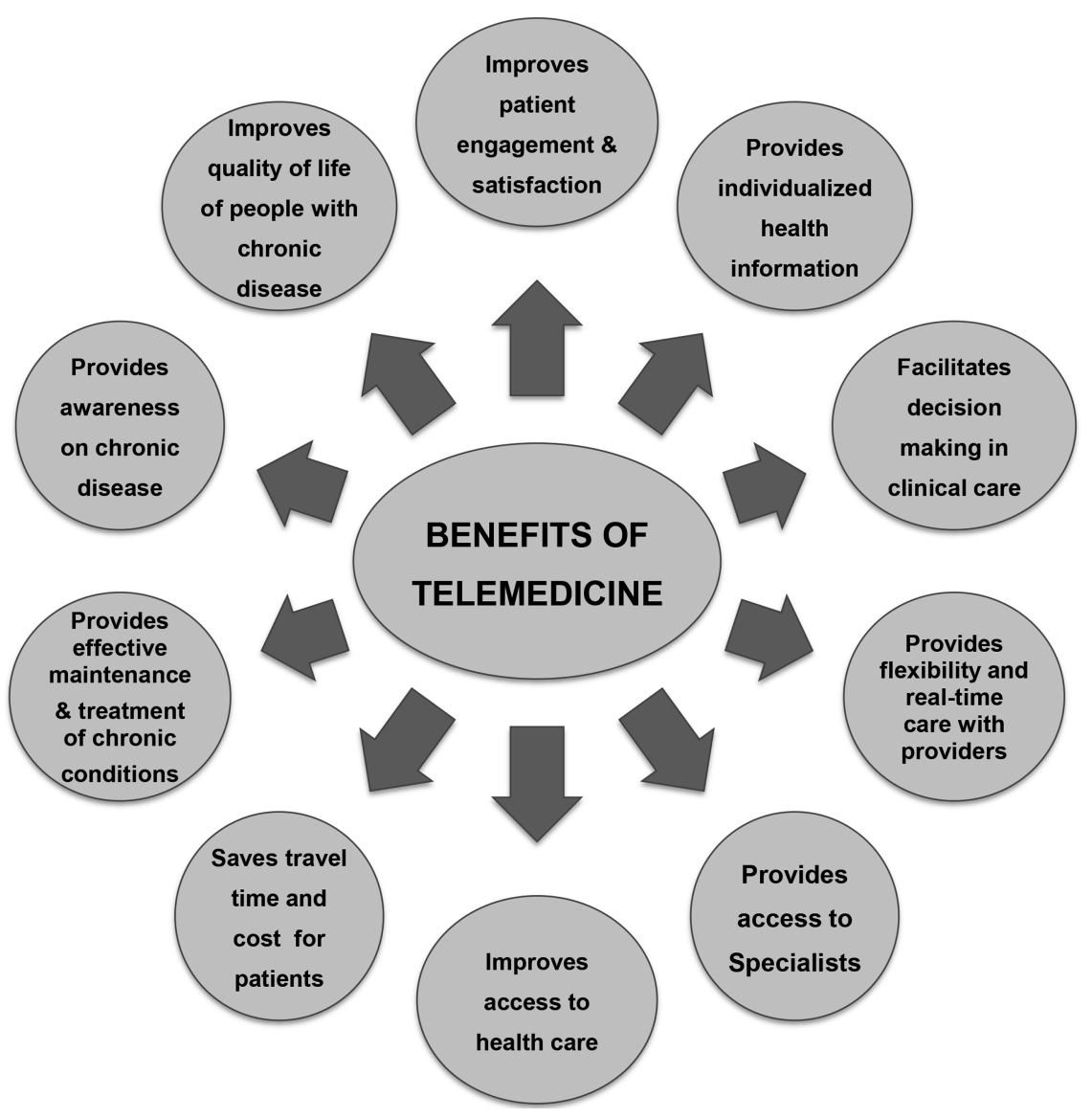

FIG. 2. Benefits of telemedicine.

automation of retinal image analysis offers opportunities to expand the telescreening services to more remote areas. ${ }^{46,47}$ AI-based diabetic retinopathy grading algorithms could potentially expedite early detection of diabetic retinopathy happening at the primary care level, and thus would be useful in reducing the burden to the health systems in screening large numbers of people with diabetes. ${ }^{45,47}$ Only people who have sight threatening/referable diabetic retinopathy would need to be referred to the ophthalmologist/retina specialist. ${ }^{45}$

\section{Benefits of telemedicine in LMIC}

The multiple benefits of telemedicine in LMIC are depicted in Figure 2. Telemedicine helps detect and manage chronic diseases such as diabetes in remote rural areas where such medical facilities are not available, accessible, and affordable. It provides individualized health information, facilitates clinical decision making, increases awareness on diabetes through education, provides easy accessibility to diabetes care at their doorsteps, and improves the quality of life of individuals with diabetes. If used effectively in remote rural areas, the telemedicine model can dramatically reduce the number of patients who need to travel to specialized centers in urban areas to get proper diagnosis and treatment, which would likely improve adherence rates.

In a 2006 study, Paul et al. conducted a survey to assess patient satisfaction levels and factors influencing satisfaction during teleophthalmology consultations in India. ${ }^{43} \mathrm{~A}$ total of 348 adults responded to the survey. Some of the main reasons respondents reported for increased satisfaction with the diabetic retinopathy telescreening were reduced travel time, lower travel costs, and easier access to clinical support systems. ${ }^{43}$ From a health care provider perspective, this program is cost-effective as it does not require the full-time services of highly qualified specialists. Several studies on cost-effectiveness have demonstrated that teleophthalmology holds great promise compared with traditional ophthalmologist examination. ${ }^{48-50}$ A systematic review of 16 studies of teleophthalmology screening for chronic eye diseases, which examined the costeffectiveness of teleophthalmology compared with traditional ophthalmologist examination, after accounting for initial capital costs, concluded that teleophthalmology is cost saving, regardless of geographical setting and with evidence of acceptability among patients and providers. ${ }^{48}$

Cost-effectiveness of teleophthalmology depends on the administrative costs of establishing and maintaining screening at regular intervals and on achieving sufficient coverage. ${ }^{50}$ In addition, teleophthalmology, compared to conventional care, has been reported to be an accurate and reliable test for detecting diabetic retinopathy and macular edema. ${ }^{51}$ Thus, telemedicine provides flexibility and real-time care with health care providers working in resource-constrained settings where, presently, this may be the only alternative.

\section{Barriers and Challenges to Telemedicine}

Although telemedicine clearly has a wide range of potential benefits, it also has some disadvantages/barriers. 
According to the World Health Organization, the biggest barrier to the implementation of telemedicine programs, globally, is the perception that the costs of setting up the telemedicine unit are too high (60\% of responding countries consider this as a barrier). ${ }^{52}$ For developing countries, high costs, underdeveloped infrastructure, and lack of technical expertise are just some challenges. ${ }^{52}$ Legal issues surrounding patient privacy and confidentiality, competing health system priorities, and a perceived lack of demand are other barriers to large-scale implementation of telemedicine. ${ }^{53}$

Some people worry about a potential breakdown in the relationship between health care provider and patient. In addition, organizational and bureaucratic difficulties are likely to be present due to lack of personal contact. A systematic review, which assessed 50 studies on telemedicine, concluded that evidence regarding the effectiveness or cost-effectiveness of telemedicine is still limited and that only a few telemedicine applications can be recommended for broader use. ${ }^{54}$

Various potential technical and structural problems could also hinder the adoption of telemedicine programs. Lack of connectivity between stand-alone diabetes telemedicine systems and hospital electronic medical records, inadequate data encryption and security systems to fully ensure patient privacy, and inadequate decision support software are some of the issues currently facing telemedicine programs, all of which obviously, need to be addressed. ${ }^{54}$

\section{Conclusion}

In low- and middle-income economies, innovative approaches, including telemedicine, mobile technology, internet, and websites, can be adapted to prevent and manage diabetes in the community. Using these technologies, clinicians can reach out to remote and underserved communities and serve patients at their doorstep.

Telemedicine is a valuable, cost-effective and reliable tool for screening people with diabetes for various complications, and it provides people in rural areas access to many virtual "super specialists" in the field of medicine. As such, it holds great promise for enhancing the quality of care, improved access to care in remote areas, and prevention and detection of noncommunicable diseases, including diabetes.

In summary, telemedicine bridges the gap between primary care and secondary/tertiary care. However, using this technology requires services of expert teams working collaboratively to provide care and follow-up. There is ample scope for telemedicine to improve diabetes care outcomes by reducing the rate of secondary complications in both developed and developing nations. Successful telemedicine-driven diabetic complication screening could reduce the risk of microvascular and macrovascular complications due to diabetes and, thereby, the health care burden.

One could anticipate that the use of automated AI software combined with telemedicine could enable efficient real-time screening of complication like retinopathy in people with diabetes, in remote areas where such facilities are currently not available.

\section{Acknowledgments}

The authors also wish to thank Christopher G. Parkin (CGParkin Communications, Inc., Henderson, NV) for edi- torial assistance. Roche Diabetes Care provided funding for the development of this article.

\section{Author Disclosure Statement}

R.P., R.R., and V.M. have no conflict of interests to disclose for this article.

\section{References}

1. International Diabetes Federation: IDF Diabetes Atlas, 8th ed. Brussels, Belgium: International Diabetes Federation, 2017.

2. Kapur A, Bjork S, Nair J, et al.: Socio-economic determinants of the cost of diabetes in India. Diabetes Voice 2004; 49:18-21.

3. Ramachandran A, Snehalatha C, Vijay V, King H: Impact of poverty on the prevalence of diabetes and its complications in urban southern India. Diabet Med 2002;19: $130-135$

4. Anjana RM, Deepa M, Pradeepa R, et al.: Prevalence of diabetes and prediabetes in 15 states of India: results from the ICMR-INDIAB population-based cross-sectional study. Lancet Diabetes Endocrinol 2017;5:585-596.

5. Donnelly R, Emslie-Smith AM, Gardner ID, Morris AD: $\mathrm{ABC}$ of arterial and venous disease: vascular complications of diabetes. BMJ 2000;320:1062-1066.

6. Unnikrishnan R, Anjana RM, Deepa M, et al.: Glycemic control among individuals with self-reported diabetes in India - the ICMR-INDIAB Study. Diabetes Technol Ther 2014;16:596-603.

7. Downey LH: Rural populations and health: determinants, disparities, and solutions book review. Prev Chronic Dis 2013;10:130097.

8. Siminerio LM: The role of technology and the chronic care model. J Diabetes Sci Technol 2010;4:470-475.

9. Wilson M: The future of telemedicine. Stud Health Technol Inform 2002;80:129-136.

10. WHO: mHealth. New Horizons for Health Through Mobile Technologies: Second Global Survey on eHealth. Geneva: World Health Organization, 2011.

11. Fjeldsoe BS, Marshall AL, Miller YD: Behavior change interventions delivered by mobile telephone short-message service. Am J Prev Med 2009;36:165-173.

12. Donner J: Research approaches to mobile use in the developing world: a review of the literature. Inf Soc 2008;24: $140-159$.

13. Travasso C: Lifestyle advice by text messages helps prevent type 2 diabetes in high risk men. BMJ 2013;347: f5750.

14. Somannavar S, Lanthorn H, Pradeepa R, et al.: Prevention awareness counselling and evaluation (PACE) diabetes project: a mega multi-pronged program for diabetes awareness and prevention in South India (PACE- 5). J Assoc Physicians India 2008;56:429-435.

15. Buis LR, Hirzel L, Turske SA, et al.: Use of a text message program to raise type 2 diabetes risk awareness and promote health behavior change (part I): assessment of participant reach and adoption. J Med Internet Res 2013;19: e281.

16. Haddad NS, Istepanian R, Philip N, et al:: A feasibility study of mobile phone text messaging to support education and management of type 2 diabetes in Iraq. Diabetes Technol Ther 2014;16:454-459. 
17. Heatley E, Middleton P, Hague W, Crowther C: The DIAMIND study: postpartum SMS reminders to women who have had gestational diabetes mellitus to test for type 2 diabetes: a randomised controlled trial-study protocol. BMC Pregnancy Childbirth 2013;12:92.

18. Ramachandran A, Snehalatha C, Ram J, et al.: Effectiveness of mobile phone messaging in prevention of type 2 diabetes by lifestyle modification in men in India: a prospective, parallel-group, randomized controlled trial. Lancet Diabetes Endocrinol 2013;1:191-198.

19. Somannavar S, Lanthorn H, Deepa $\mathbf{M}$, et al.: Increased awareness about diabetes and its complications in a whole city: effectiveness of the "prevention, awareness, counselling and evaluation" PACE Diabetes Project PACE-6. J Assoc Physicians India 2008;56:497-502.

20. Garg SK, Shah VN, Akturk HK, et al.: Role of mobile technology to improve diabetes care in adults with type 1 diabetes: the remote-T1D Study iBGStar in type 1 diabetes management. Diabetes Ther 2017;8:811-819.

21. Miller KM, Beck RW, Bergenstal RM, et al.: Evidence of a strong association between frequency of self-monitoring of blood glucose and hemoglobin A1c levels in T1D exchange clinic registry participants. Diabetes Care 2013;36:20092014.

22. Harashima S, Nishimura A, Ikeda K, et al.: Once daily selfmonitoring of blood glucose (SMBG) improves glycemic control in oral hypoglycemic agents (OHA)-treated diabetes: SMBG-OHA follow-up study. J Diabetes Sci Technol 2015;10:378-382.

23. Polonsky WH, Fisher L, Schikman CH, et al.:. A structured self-monitoring of blood glucose approach in type 2 diabetes encourages more frequent, intensive, and effective physician interventions: results from the STeP study. Diabetes Technol Ther 2011;13:797-802.

24. Quinn CC, Clough SS, Minor JM, et al.: WellDoc mobile diabetes management randomized controlled trial: change in clinical and behavioral outcomes and patient and physician satisfaction. Diabetes Technol Ther 2008;10:160168.

25. Quinn CC, Shardell MD, Terrin ML, et al.: Clusterrandomized trial of a mobile phone personalized behavioral intervention for blood glucose control. Diabetes Care 2011; 34:1934-1942.

26. Mora P, Buskirk A, Lyden M, et al: Use of a novel, remotely connected diabetes management system is associated with increased treatment satisfaction, reduced diabetes distress, and improved glycemic control in individuals with insulin-treated diabetes: first results from the Personal Diabetes Management Study. Diabetes Technol Ther 2017; 19:715-722.

27. Overland J, Abousleiman J, Chronopoulos A, et al.: Improving self-monitoring of blood glucose among adults with type 1 diabetes: results of the Mobile ${ }^{\mathrm{TM}}$ Study. Diabetes Ther 2014;5:557-565.

28. Dasgupta A, Deb S: Telemedicine: a new horizon in public health in India. Indian J Community Med 2008;33:3-8.

29. Thomas R, Paul P, Rao GN, et al.: Present status of eye care in India. Surv Ophthalmol 2005;50:85-101.

30. Schoenfeld ER, Greene JM, Wu SY, Leske MC: Patterns of adherence to diabetes vision care guidelines: baseline findings from the Diabetic Retinopathy Awareness Program. Ophthalmology 2001;108:563-571.

31. Vashist P, Singh S, Gupta N, Saxena R: Role of early screening for diabetic retinopathy in patients with diabetes mellitus: an overview. Indian J Community Med 2011;36: 247-252.

32. Namperumalsamy P, Nirmalan PK, Ramasamy K: Developing a screening program to detect sight-threatening diabetic retinopathy in South India. Diabetes Care 2003;26: 1831-1835.

33. Moss SE, Klein R, Kessler SD, Richie KA: Comparison between ophthalmoscopy and fundus photography in determining severity of diabetic retinopathy. Ophthalmology 1985;92:62-67.

34. Surendran TS, Raman R: Teleophthalmology in diabetic retinopathy. J Diabetes Sci Technol 2014;8:262-266.

35. Mohan V, Deepa M, Pradeepa R, et al.: Prevention of diabetes in rural India with a telemedicine intervention. J Diabetes Sci Technol 2012 1;6:1355-1364.

36. Liesenfeld B, Kohner E, Piehlmeier W, et al.: A telemedical approach to the screening of diabetic retinopathy: digital fundus photography. Diabetes Care 2000;23:345348.

37. Li HK, Horton M, Bursell SE, et al.: Telehealth practice recommendations for diabetic retinopathy, second edition. Telemed J E Health 2011;17:814-837.

38. Digital Imaging and Communications in Medicine (DICOM): The DICOM Standard. www.dicomstandard.org/ current (accessed January 10, 2019).

39. Rajalakshmi R, Arulmalar S, Usha M, et al.: Validation of smartphone based retinal photography for diabetic retinopathy screening. PLoS One 2015;24:e0138285.

40. Wilkinson CP, Ferris FL 3rd, Klein RE, et al.: Proposed international clinical diabetic retinopathy and diabetic macular edema disease severity scales. Ophthalmology 2003; 110:1677-1682.

41. Gomez-Ulla F, Alonso F, Aibar B, Gonzalez F: A comparative cost analysis of digital fundus imaging and direct fundus examination for assessment of diabetic retinopathy. Telemed J E Health 2008;14:912-918.

42. Kumar S, Yogesan K, Hudson B, Tay-Kearney ML: Internet based electronics eye care consultations: Patient perspective. In: Yogesan K, Kumar S, Goldschmidth L, Cuadros J, eds. Teleopthhthalmology. Springer: Verlag, 2008, pp. 133-138.

43. Paul PG, Raman R, Rani PK, et al.: Patient satisfaction levels during teleophthalmology consultation in rural South India. Telemed J E Health 2006;12:571-578.

44. Gulshan V, Peng L, Coram M, et al.: Development and validation of a deep learning algorithm for detection of diabetic retinopathy in retinal fundus photographs. JAMA 2016;316:2402-2410.

45. Rajalakshmi R, Subashini R, Anjana RM, Mohan V: Automated diabetic retinopathy detection in smartphonebased fundus photography using artificial intelligence. Eye (London) 2018;32:1138-1144.

46. Das T, Raman R, Ramasamy K, Rani PK: Telemedicine in diabetic retinopathy: current status and future directions. Middle East Afr J Ophthalmol 2015;22:174-178.

47. Kapetanakis VV, Rudnicka AR, Liew G, et al.: A study of whether automated Diabetic Retinopathy Image Assessment could replace manual grading steps in the English National Screening Programme. J Med Screen 2015;22: 112-118.

48. Sharafeldin N, Kawaguchi A, Sundaram A, et al.: Review of economic evaluations of teleophthalmology as a screening strategy for chronic eye disease in adults. $\mathrm{Br} \mathrm{J}$ Ophthalmol 2018;102:1485-1491. 
49. de la Torre-Díez I, López-Coronado M, Vaca C, et al.: Cost-utility and cost-effectiveness studies of telemedicine, electronic, and mobile health systems in the literature: a systematic review. Telemed J E Health 2015;21:81-85.

50. Rachapelle S, Legood R, Alavi Y, et al. The cost-utility of telemedicine to screen for diabetic retinopathy in India. Ophthalmology 2013;120:566-573.

51. Whited JD: Accuracy and reliability of teleophthalmology for diagnosing diabetic retinopathy and macular edema: a review of the literature. Diabetes Technol Ther 2006;8: 102-111.

52. WHO: Telemedicine: opportunities and developments in Member States: report on the second global survey on eHealth 2009. www.who.int/goe/publications/goe_telemedicine_ 2010.pdf (accessed March 11, 2015).

53. Roine R, Ohinmaa A, Hailey D: Assessing telemedicine: a systematic review of the literature. CMAJ 2001;165: $765-771$.
54. Klonoff DC: Using telemedicine to improve outcomes in diabetes - an emerging technology. J Diabetes Sci Technol 2009;3:624-628.

Address correspondence to: Viswanathan Mohan, MD, FRCP, PhD, $D S c, F A C P, F A C E, M A C P$ Madras Diabetes Research Foundation and Dr. Mohan's Diabetes Specialties Centre WHO Collaborating Centre for Non-communicable Diseases Prevention and Control IDF Centre of Excellence in Diabetes Care No. 4, Conran Smith Road Gopalapuram, Chennai 600086 India

E-mail: drmohans@diabetes.ind.in 